\title{
Brasseur, Guy P.; Jacob, Daniela; Schuck-Zöller, Susanne (Hrsg.) (2017): Klimawandel in Deutschland. Entwicklung, Folgen, Risiken und Perspektiven
}

\author{
Berlin/Heidelberg: Springer Spektrum. 348 S., 116 Abb.
}

\section{Barbara Warner ${ }^{1}$ (D)}

Online publiziert: 29. Mai 2017

(C) Springer-Verlag Berlin Heidelberg 2017

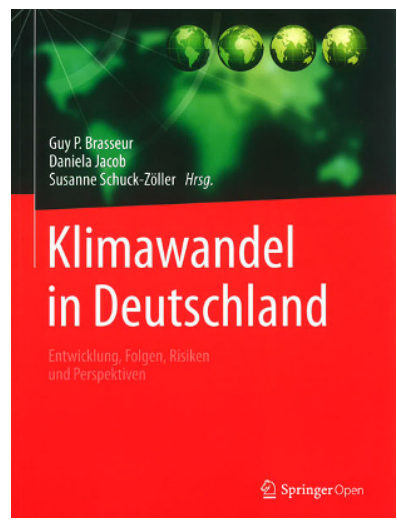

Die Autoren dieses aktuellen Bandes reflektieren den Klimawandel in Deutschland aus einer umfassenden Perspektive. Dass die Unsicherheiten bezüglich valider Aussagen zu den Folgen des Klimawandels bereits an einer sehr frühen Stelle behandelt werden, spiegelt die große Herausforderung für Politik, Wirtschaft, Gesellschaft und auch für die räumliche Planung wider (zu grundsätzlichen gesellschaftlichen und wirtschaftlichen Herausforderungen vgl. z. B. Karczmarzyk/Pfriem 2011; WBGU 2014; Roser/Seidel 2015). Die Notwendigkeit und die Schwierigkeit, vielfältige räumliche und zeitliche Skalen bei der Vorbereitung konkreter Adaptions- oder Mitigationsmaßnahmen berücksichtigen zu müssen, beschreiben unter anderem Knieling und Müller (2015) sowie Renn (2016). Im vorliegenden Werk wird zielführend, knapp und trotzdem umfassend

Dr. Barbara Warner

warner@arl-net.de

1 Akademie für Raumforschung und Landesplanung, Leibniz-Forum für Raumwissenschaften, Hohenzollernstraße 11, 30161 Hannover, Deutschland auf die Kernpunkte der Schutz- und Anpassungsmaßnahmen auch im kleinräumigen Maßstab eingegangen und ihre Bedeutung für die gesellschaftliche Transformation herausgestellt.

Mit einer Einordnung der Bedeutung (globaler) Klimamodelle und Szenarien gehen die Herausgeber gleich zu Beginn auf methodische Anforderungen ein, aus Modellen und Szenarien konkrete Handlungsoptionen abzuleiten. Der Einordnung des Oberthemas Klimawandel in den globalen Zusammenhang folgen knapp zusammengefasst Aussagen zu Deutschland insgesamt bis hin zur regionalen Ebene und ermöglichen so anwendungsorientierte Zugänge zu dem komplexen Thema. Ebenfalls im ersten Hauptkapitel betonen beispielsweise Andreas Dobler, Hendrik Feldmann und Uwe Ulbrich die Schwierigkeit, bei Unsicherheiten bezüglich konkreter Klimaauswirkungen Modelle entwickeln zu können. Sie sensibilisieren für diese methodologischen Hürden für Wissenschaft bzw. Anwendung, indem sie der Modellierung einen prominenten Platz einräumen. Regionale Klimamodelle erlauben zwar aufgrund regionaler Wirkungszusammenhänge durchaus detaillierte Darstellungen, stoßen aber bereits aufgrund der relativ hohen Auflösung und der zunehmenden Abhängigkeit der berücksichtigten Faktoren von konkreten regionalen Phänomenen an Grenzen hinsichtlich möglichst valider Aussagen. Auch kleinräumige Wechselwirkungen wie zum Beispiel zwischen Boden, Küsten, Topographie und Atmosphäre müssen für annähernd verlässliche Aussagen erfasst und einbezogen werden. Wenn im Folgenden die Faktoren Temperatur, Niederschlag, Wind und Wasserregime detailliert mit regionalen Spezifika und (erwartbaren) Trends beschrieben werden, wird so immer wieder auf die genannten Unsicherheiten bei möglichen methodischen Zugängen geschlossen.

Fakten und prognostische Aussagen für Deutschland liefert das zweite Hauptkapitel. Die umfassende Übersicht 
über die wesentlichen Klimaparameter und ihre Veränderungen bietet einen großen Mehrwert für alle, die sich einen Überblick über Trends und auch über konkrete regionale Auswirkungen verschaffen wollen. Hochwasserereignisse nehmen in diesem Kapitel einen besonderen Raum ein. Dies ist der hohen gesellschaftlichen Relevanz dieser Extremereignisse zuzurechnen. Zahlreiche Initiativen, grenzüberschreitend und im (auch internationalen) Dialog entwickelt, zeigen die große auch gesellschaftspolitische Bedeutung dieses Themas. Kapitel 10.1 etwa liefert Aussagen zu Abflussdaten für einzelne Flusseinzugsgebiete in Deutschland. Allein hierdurch wird es unter anderem kommunalen Akteuren ermöglicht, sich einen Überblick über Modellierungsansätze in konkreten Räumen zu verschaffen. Ein wichtiger Denkanstoß für alle, die sich mit Klimaschutzmaßnahmen auseinandersetzen, ist auch Kapitel 11 als Exkurs zum Thema Unsicherheiten in der Entscheidungsfindung, das zum Ende des Buches wieder aufgegriffen wird. Der Autor dieses Kapitels (Manfred Mudelsee) beschreibt die Schwierigkeit, sich bereits durch die Fragestellung für eine Analyse - exemplarisch von Eintrittswahrscheinlichkeiten - auf ein unsicheres Terrain zu begeben. Die folgende Auseinandersetzung mit Naturgefahren wie Massenbewegungen verdeutlicht zudem, dass eindeutige Zuordnungen von klimawandelbedingten Ursachen zu Schadereignissen komplex bzw. manchmal unmöglich sind: Welche Auswirkungen sind auf direkte menschliche Eingriffe, welche auf (schleichende) klimatische Veränderungen zurückzuführen? Die fundiert begründeten Darstellungen von Eintrittswahrscheinlichkeiten, knapp gehaltene Interpretationen und das Fehlen von Wertungen und Zuspitzungen lassen Raum, um eigene Schlüsse zu ziehen.

Denken in thematischen statt in räumlichen Zusammenhängen wird angeregt, indem im dritten Hauptkapitel auf die Auswirkungen des Klimawandels eingegangen wird. Die Herausgeber verdeutlichen deren Komplexität in einem sehr heterogen strukturierten und infrastrukturell geprägten Land wie Deutschland anhand ausgewählter Schwerpunkte. Die Themen Verkehr, Land- und Forstwirtschaft oder Tourismus werden ebenso angesprochen wie die Entwicklung von Biodiversität und der Bereich Gesundheit. In den Unterkapiteln werden konkrete Maßnahmen bewertet. Die Relevanz des Oberthemas Klimawandel für Entscheidungswege in Politik, Gesellschaft und Planung wird hier hervorgehoben. Der Aufbau der Kapitel macht dabei gut nachvollziehbar, wie schwierig konkrete Strategien umzusetzen sind und welche Entwicklungs- oder Schutzmöglichkeiten etwa für Infrastrukturen oder für die Biodiversität bestehen. Bei einigen Sparten finden sich Hinweise für Planungsträger (Kapitel 16) oder für die Politik (Kapitel 14 und 15). Das Thema Boden (Kapitel 20) hebt die Bedeutung des Klimawandels für die Landökosysteme hervor, bevor zum Themenkomplex der Infrastrukturen (Verkehr, Städte, Energie- und Wasserversorgung) übergeleitet wird (Kapitel 21, 22 und 24).

Städte als vom Klimawandel stark betroffene Lebensräume bekommen ein eigenes Unterkapitel, in dem Herausforderungen einer klimaangepassten Stadtentwicklung und einer angemessenen Governance zentral sind. Über Bezüge zur baulichen Umwelt und zur Bevölkerung werden Zusammenhänge zu den Vorläuferkapiteln ermöglicht. Die Notwendigkeit einer Multilevel Governance in Städten bei gegebenen administrativen Strukturen ist eine große Aufgabe. Die Komplexität des Themas zeigt sich unter anderem an den Anforderungen an die Energie- und Wasserversorgung (Kapitel 24). Veränderte Niederschlagsregime oder Nachfragemuster im Stromverbrauch verdeutlichen beispielsweise die Notwendigkeit von flexiblen Lösungen, die den demographischen, siedlungsstrukturellen und wirtschaftlichen Wandel berücksichtigen können. Flexible Anpassungsstrategien sind auch im Tourismus (Kapitel 23) notwendig, um mit sich ändernden Voraussetzungen für den Tourismus selbst (abnehmende Schneesicherheit) oder mit (wiederkehrenden) Extremereignissen wie Hitzewellen umzugehen.

Vor der Benennung der möglichen Kosten des Klimawandels werden in Kapitel 4 die übergreifenden Risiken und Unsicherheiten dargelegt. Die Kosten der genannten systemischen Veränderungen sind direkt abhängig vom gesellschaftlichen Verhalten, das das wirtschaftliche Ausmaß des Klimawandels steuert. Es ist sehr gut nachvollziehbar, dass Größen wie Wirtschaftsleistung oder Einkommen nur unter unsicheren Annahmen in Szenarien eingespeist werden können. Indirekte Effekte, immaterielle Schäden bleiben in der Regel ausgeklammert. Somit sind beispielsweise strategisch-politische Rahmenbedingungen, Werte und Vulnerabilitäten wesentliche Einflussfaktoren auf Klimaauswirkungen.

Das vierte Hauptkapitel thematisiert somit vielschichtig die gesellschaftlichen Dimensionen des Klimawandels anhand internationaler und nationaler Strategien. Sozioökonomische Entwicklungspfade stellen wesentliche Parameter dar, die Klimaeffekte bestimmen. Chancen und Risiken für Anpassungsprozesse sollten daher aus der Perspektive des Klimawandels und aus der gesellschaftlichen Perspektive diskutiert werden - auch wenn letztendlich Ungewissheit in Planungs- und Entscheidungsprozessen als ,zusätzliche Dimension der Unsicherheit" (S. 275) verbleiben wird. Dass die Fülle an Studien und Modellen teilweise die Zugänge zu konkreten Themenkomplexen erschwert, greifen die Autoren (Mark Fleischhauer, Stefan Greiving, Christian Lindner, Johannes Lückenkötter und Inke Schauser) in Kapitel 27 (Literaturanalyse) auf. In einer Zusammenschau von Vulnerabilitätsanalysen wurden die wichtigsten Klimawirkungen handlungsfeldübergreifend analysiert.

Jürgen Scheffran setzt sich zum Beispiel mit dem potenziellen Konfliktpotenzial des Klimawandels in komplexen, 
explizit politischen Systemen auseinander (S. 287 ff.). Entwicklungen mit globalen Maßstäben wie klimabedingte Migration und politische Instabilität in vulnerablen Regionen sind hier elementar. Die Folgen des Klimawandels verstärken sich in gesellschaftlichen Systemen, können politische Stabilität untergraben und Krisen verursachen. Das Thema wird weitergeführt hin zur Kommunikation und Steuerung von Risiken (Kapitel 29). Für planende und politische Akteure ist es elementar zu wissen, wie Risiken kommuniziert werden können und wie mit Zielkonflikten umgegangen werden kann (S. 299). Über ein „Risk-Governance-Modell“ des Internationalen Risikorates (IRGC) können unter anderem Klimarisiken an der Schnittstelle zwischen Wissenserzeugung und Entscheidung/Umsetzung besser abschätzbar gemacht werden. Darüber hinaus entscheiden Politik und Verwaltung in (komplexen) gesellschaftlichen Systemen, welche auch normativen Vorgaben den Umgang mit Unsicherheiten bestimmen - auf der Basis von wissenschaftlich erarbeiteten Vorschlägen, die etwa über Szenarien die Politikberatung prägen. Somit plädieren die Autoren für einen frühzeitigen Dialog mit gesellschaftspolitischen Akteuren, in dem Unsicherheiten im Umgang mit Klimafolgen benannt werden müssen. So kann ein Grundkonsens für nachhaltige Entscheidungen gefunden werden. Die Herausgeber schlagen somit einen weiten Bogen über konkrete Klimafolgen und methodische Ansätze zu politischen Herausforderungen und letztendlich zur gesellschaftlichen Transformation: „Soziale Innovationen“ tragen im Rahmen einer Anpassung an den Klimawandel zur nachhaltigen Entwicklung bei (S. 341).

Das Werk ist als Fundus für Fakten, Zusammenhänge, Strategien und Argumentationsgrundlagen elementar wichtig. Auch wenn der umfassende Themenkomplex des Klimawandels in Deutschland anhand ausgewählter Schwerpunkte beleuchtet wird, erschließen sich Zusammenhänge und Folgewirkungen leicht. Die übersichtliche Struktur der
Kapitel trägt sehr zur Lesbarkeit und auch zur Einprägsamkeit bei. Verständlich aufbereitet werden die wesentlichen Zusammenhänge und Studien erläutert und priorisiert. Wenn Rückschlüsse auf konkret raumrelevante Maßnahmen nicht gelingen (wollen), erfolgt eine fundierte Darstellung der Grundlagen und eventuell weiterführender wissenschaftlicher Zugänge. Folgerichtig fasst das letzte Kapitel zu übergreifenden Risiken und Unsicherheiten die systemischen Aussagen zusammen.

„In Deutschland trägt die Gesellschaft Verantwortung für den nachhaltigen Umgang mit den natürlichen Ressourcen Boden, Wasser und Luft" (S. 211) - diese Aussage macht den universellen und komplexen Kern aller Maßnahmen und die Zuständigkeit Aller deutlich. Dass dieser Umgang nicht nachhaltig ist, ist ein Kernproblem der Klimaschutzdebatte. Nur mit veränderten Lebensstilen und Konsummustern, neuen Wohlfahrts- und Wertesystemen kann eine „klimaresiliente Gesellschaft angestoßen, umgesetzt und verstetigt werden“ (S. 321). Hierzu befähigt das vorliegende Werk alle Akteure, die sich mit dem Klimawandel in Deutschland auseinandersetzen.

\section{Literatur}

Karczmarzyk, A.; Pfriem, R. (Hrsg.) (2011): Klimaanpassungsstrategien von Unternehmen. Marburg. = Theorie der Unternehmung 51.

Knieling, J.; Müller, B. (Hrsg.) (2015): Klimaanpassung in der Stadtund Regionalentwicklung. Ansätze, Instrumente, Maßnahmen und Beispiele. München.

Renn, O. (2016): Inclusive Resilience: A New Approach to Risk Governance. In: IRGC - International Risk Governance Council (Hrsg.): Resource Guide on Resilience. Lausanne, 1-8.

Roser, D.; Seidel, C. (2015): Ethik des Klimawandels. Eine Einführung. Darmstadt.

WBGU - Wissenschaftlicher Beirat der Bundesregierung Globale Umweltveränderungen (2014): Klimaschutz als Weltbürgerbewegung. Sondergutachten. Berlin. 